\title{
The Impact of Valenced Verbal Information on Implicit and Explicit Evaluation: The Role of Information Diagnosticity, Primacy, and Memory Cueing
}

\author{
Pieter Van Dessel ${ }^{\mathrm{a} *}$, Jeremy Cone ${ }^{\mathrm{b}}$, Anne Gast ${ }^{\mathrm{c}}$, and Jan De Houwer ${ }^{\mathrm{a}}$ \\ aDepartment of Experimental-Clinical and Health Psychology, Ghent University, Ghent, Belgium; \\ ${ }^{\mathrm{b}}$ Department of Psychology, Williams College, Williamstown, United States; ${ }^{\mathrm{c} D e p a r t m e n t ~ o f ~}$ \\ Psychology, University of Cologne, Cologne, Germany
}

This paper is not the copy of record and may not exactly replicate the final, authoritative version of the article. Please do not copy or cite without authors permission. The final article will be available, upon publication, via its DOI: 10.1080/02699931.2019.1594703

\section{Authors' Note}

The research reported in this paper was funded by the Scientific Research Foundation, Flanders under Grant FWO16/PDO/201 to PVD, by the Deutsche Forschungsgemeinschaft under grant GA 1520/2-1 to AG and by the Ghent University Methusalem Grant BOF09/01M00209 to JDH. There was no potential conflict of interest to report. Correspondence concerning this article should be sent to PVD, Ghent University, Department of Experimental-Clinical and Health Psychology, Henri Dunantlaan 2, B-9000 Ghent (Belgium). E-mail: Pieter.vanDessel@UGent.be. 


\begin{abstract}
Previous research has shown that the presentation of valenced information about a target stimulus sometimes has different effects on implicit and explicit stimulus evaluations. Importantly, however, research examining the moderators of implicit-explicit dissociations has often failed to account for differences in the properties of the instruments used to measure implicit and explicit evaluations, preventing a clear interpretation of the results. In an effort to overcome these limitations, we conducted a study that probed the impact of valenced information on implicit and explicit evaluations as measured with procedures that were matched on methodological factors. Participants first read positive and negative information about a person named Bob and then completed measures of implicit and explicit evaluations of Bob. We examined the moderating effect of three characteristics: information diagnosticity, primacy, and whether information retrieval was cued during evaluation. Results of two high-powered experiments showed an effect of diagnosticity on implicit and explicit evaluations, replicating previous work, and extending it to new evaluation measures. We observed primacy effects on explicit evaluations in Experiment 1 and on implicit evaluations in Experiment 2. However, we did not observe memory cueing effects or any interactions. We discuss practical implications as well as implications for cognitive evaluation theories.
\end{abstract}

Keywords: implicit evaluation, implicit-explicit dissociation, structural fit, diagnosticity, memory cueing, primacy 


\section{The Impact of Valenced Verbal Information on Implicit and Explicit Evaluation: The Role of Information Diagnosticity, Primacy, and Memory Cueing}

The extent to which people evaluate stimuli as good or bad has long been a central research focus in psychology (Allport, 1935). Contemporary research on this topic often involves a comparison of more automatic or implicit evaluations with less automatic or explicit evaluations (Gawronski \& Brannon, 2018). Although the term "evaluation" is often used to refer to mental constructs (i.e., attitudinal representations), we use this term to refer to a behavioral phenomenon, that is, the impact of stimuli on evaluative responses. This allows for a clearer distinction between the behavior that needs to be explained (i.e., evaluation) and explanatory construct (e.g., evaluative associations in memory; De Houwer, Gawronski, \& Barnes-Holmes, 2013). The distinction between implicit and explicit evaluation then refers to the fact that evaluation sometimes occurs under conditions of automaticity (i.e., uncontrolled, unconscious, efficient, or fast: Moors \& De Houwer, 2006). Implicit evaluations are automatic in the sense that they exhibit automaticity features. An important reason for exploring (distinctions between) implicit and explicit evaluations is that some studies have provided evidence that these two types of evaluation can differentially relate to behavior (see Cameron, Brown-Iannuzzi, \& Payne, 2012; Friese, Hofmann, \& Schmidt, 2008, for reviews). For instance, compared to explicit evaluations, implicit evaluations are sometimes better predictors of other types of behaviors that are also emitted under (some of) the conditions of automaticity (e.g., unintentional nonverbal expression; e.g., Banaji \& Greenwald, 2013).

When trying to understand implicit and explicit evaluation, an important question is how people learn these different types of evaluation. Research on this topic has mainly focused on examining the differential responsiveness of implicit and explicit evaluations to different kinds of 
valenced information (e.g., verbal, pictorial) and the characteristics of this information (e.g., consistency, primacy). Sometimes dissociations are observed in this research (i.e., observed differences between evaluations that have different features of automaticity) (e.g., Gregg, Seibt, \& Banaji, 2016). Many studies have used a set-up in which participants first received valenced information about unknown target stimuli and are then probed for resulting changes in their more automatic (implicit) and less automatic (explicit) evaluations of these stimuli (see Gawronski \& Brannon, 2018, for an overview). Importantly, however, there is little unambiguous empirical evidence about the factors that (differentially) impact implicit and explicit evaluation. This is largely the result of three important limitations of research on this topic. First, many of the (initial) studies that tested moderators of implicit and explicit evaluation learning had low statistical power and it is therefore unclear whether observed (sometimes dissociative) effects are robust and replicable (see Heycke, Gehrmann, Haaf, \& Stahl, 2018, for a discussion). Second, though several studies have directly compared effects of a single moderator of evaluative learning (e.g., information diagnosticity) on implicit and explicit evaluation, only few have compared effects of multiple moderators (see Rydell \& McConnell, 2006, Hu, Gawronski, \& Balas, 2017, for exceptions). As a result, it is often unclear how moderators relate to one another and how they differentially determine implicit and explicit evaluation.

A third and arguably the most important limitation is that measurement-related issues have complicated (comparative) research on implicit and explicit evaluation since its introduction. As many authors have argued, implicit evaluation measures (or constructed scores based on these responses) do not directly reflect the construct of implicit evaluation (e.g., Payne, Burkley, \& Stokes, 2008) and it is often unclear to what extent (or in what way) evaluative responses in implicit evaluation measures are in fact implicit or automatic (i.e., what automaticity features they have) 
(Gawronski \& De Houwer, 2014). Another aspect of the measurement problem involves the structural fit of implicit and explicit measures (Payne et al., 2008). When comparing implicit and explicit evaluations, there are often many procedural differences between measures (e.g., control stimuli, score calculations, or instructions) that might confound the observation of differential effects. To put it differently, the implicit and explicit measures that are compared often differ not only with regard to the implicitness of the measure, but also with regard to other factors. As a result, implicit measures are known to be sensitive to factors unrelated to evaluation, such as asymmetries in the salience of stimuli (Rothermund \& Wentura, 2004) or extra-personal knowledge (Olson \& Fazio, 2004). These and other confounding factors mean that dissociations between implicit and explicit evaluation often cannot be readily interpreted. To deal with this limitation, Payne and colleagues (2008) proposed to measure implicit and explicit evaluations in similar tasks that only differ from one another with regard to the automaticity conditions of the elicitation of evaluative responses.

The aim of the current study is to provide rich information about three important moderators of implicit and explicit evaluations while implementing the recommendations by Payne et al. (2008) to control for measurement-related structural fit issues. To this end, we employed two often used implicit evaluation measures (the Affect Misattribution Procedure, AMP: Payne, Cheng, Govorun, \& Stewart, 2005, and the evaluative priming task, EPT: Fazio, Sanbonmatsu, Powell, \& Kardes, 1986) as well as an adaption of these measures that measures explicit evaluation in a similar procedural setup (explicit AMP and explicit EPT). These two types of measures differed from one another in the provided instructions which stated that participants should either ignore the evaluative stimulus and rate their liking of another stimulus (Chinese ideographs or a valenced word) (implicit evaluation task) or rate their liking of the evaluative stimulus and ignore the other stimulus (explicit evaluation task). It is thus reasonable to argue that evaluation of the prime stimulus was more intentional in the 
latter task than in the former task. We tested effects of three manipulations that are sometimes found to have a differential effect on implicit and explicit evaluation. Specifically, we provided participants with two pieces of valenced information (one positive and one negative) about a novel person (Bob) and we examined to what extent implicit and explicit evaluations of Bob depended on (1) primacy of the information, that is, whether the positive or negative information was given first or second (Gregg et al., 2006), (2) diagnosticity of the information, that is, whether the positive or negative information was high or low in diagnosticity for making evaluative inferences (Cone \& Ferguson, 2015), and (3) memory cueing of the information, that is, whether a cue for retrieval of the positive or negative information was present during evaluation (Van Dessel, Cone et al., 2018). We included a sufficiently large number of participants to allow a high-powered test of (1) whether previously observed effects of the three information characteristics on implicit and explicit evaluation are robust (to the extent that they can be picked up with the measures) and (2) whether implicit and explicit evaluations are differentially sensitive to effects of the information characteristics.

Though the main aim of our study was to provide new, robust information about the moderators of implicit and explicit evaluation (to facilitate practical and theoretical advance), we also had a secondary aim of testing specific predictions of specific mental process accounts of evaluation. We therefore pre-specified predictions of a dual-process account (Rydell \& McConnell, 2006) and an inferential account (Van Dessel, Hughes \& De Houwer, 2018) of evaluation. Most dual-process theories of evaluation, and the account by Rydell et al. specifically, have posited that implicit evaluation involves the automatic activation of associative links in memory that cannot be readily erased on the basis of new (counter-attitudinal) information whereas explicit evaluation depends on more controlled processes such as the validation of all activated information. From this perspective, it is often assumed that the modification of implicit evaluations requires repeated pairings of target 
stimuli and valenced events as for example in evaluative conditioning procedures (e.g., De Houwer, Thomas, \& Baeyens, 2001; Hofmann, De Houwer, Perugini, Baeyens, \& Crombez, 2011) that might produce gradual changes in mental associations over time.

Importantly, however, several recent studies have found that implicit evaluations are not always resistant to rapid change (Cone \& Ferguson, 2015; Mann \& Ferguson, 2015, 2017). In fact, the presentation of new (verbal) information can sometimes even have a stronger effect on implicit than on explicit evaluations (e.g., Van Dessel, De Houwer, Gast, Smith, \& De Schryver, 2016). Furthermore, two recent reviews challenged the idea that dissociations between implicit and explicit evaluations are the result of a differential sensitivity of these types of evaluations to particular types of learning. Specifically, compared to explicit evaluations, implicit evaluations do not seem to be (1) more sensitive to repeated pairings of stimuli and valenced events (Corneille \& Stahl, 2018) and (2) less sensitive to manipulations that target reasoning processes such as information validation (Cone, Mann, \& Ferguson, 2017). On the basis of these findings, several theorists have recently questioned the idea that implicit and explicit evaluations are supported by two different types of mental processes and that observed dissociations reflect differences in these underlying processes (e.g., De Houwer, 2014; Mandelbaum, 2016). For instance, an inferential theory of evaluation postulates that the inference of evaluative information on the basis of information that is available in memory underlies both implicit and explicit evaluations (Van Dessel, De Houwer, \& Hughes, 2018). From this perspective, implicit-explicit dissociations might arise because implicit and explicit evaluation measures provide differential motivation and opportunity to integrate certain information in evaluative inferences. Specifically, explicit evaluation measures typically provide stronger incentives to engage in extensive retrieval and validation of available evaluative information about a target stimulus (e.g., because they include instructions to rate personal liking of this stimulus). In contrast, 
implicit evaluation measures might facilitate more automatic (e.g., fast or efficient) evaluative inferences that only integrate information that is easy to retrieve. For instance, they might reflect quick spontaneous inference of the valenced properties of a stimulus on the basis of the information that is most easily accessible at the time of evaluation. In support of this idea, a recent study indicated that the ease of memory retrieval of evaluative information might moderate effects of this information on implicit evaluation (Van Dessel, Cone, Gast, \& De Houwer, 2018). In that study, participants first saw valenced behavioral statements about two unfamiliar persons (i.e., Bob and Jake) together with an image that related to the behavior. Presentation of this image during evaluation (assumed to make the congruent information more easily accessible in memory) consistently influenced implicit evaluations of Bob and Jake as measured with an AMP but not explicit evaluations as measured with a rating task.

On the basis of the inferential account, we predicted that (1) higher diagnosticity, (2) primacy, and (3) memory cueing of valenced information should all make it more likely that participants will draw inferences about evaluative properties of Bob on the basis of this information and should therefore bias implicit and explicit evaluations in line with the valence of this information. We also predicted that information diagnosticity should have a stronger impact on implicit and explicit evaluations than the other moderators because diagnosticity is directly related to whether and with which certainty evaluative information can be inferred. In contrast, primacy and cueing might only facilitate memory retrieval of the valenced information, which only indirectly facilitates evaluative inferences on the basis of this information. A third prediction was that the effect of diagnosticity should be stronger on explicit than on implicit evaluation because the instruction to rate the valence of Bob (in the explicit evaluation measure) facilitates more extensive validation of relevant evaluative information on the basis of diagnosticity. We also specified a fourth prediction, that, in 
contrast to the effect of diagnosticity, the effect of memory cueing would not be bigger on explicit than on implicit evaluation. Our rationale was that cued information might be rejected as a valid basis for evaluation especially when there is a stronger focus on validation during evaluation (in the explicit evaluation measure).

On the other hand, the dual-process account of Rydell et al. (2006) would predict that (1) a primacy effect should be observed on implicit but not explicit evaluations (because, once established, associations underlying implicit evaluations might be difficult to change; see Gregg et al., 2006) and (2) a diagnosticity effect should be observed on explicit but not on implicit evaluations of Bob.

\section{Experiment 1}

\section{Participants}

A total of 297 English-speaking volunteers participated online via the Prolific Academic website (https://prolific.ac). The experiment was programmed in Inquisit 4.0 and hosted via Inquisit Web (Millisecond Software, Seattle, WA). The sample size was determined on the basis of an a priori power analysis to allow sufficient power (i.e., power $>0.90$ ) to detect small effects. Prior to data-collection, target sample size was pre-registered together with the study design, data-analysis plan and the described hypotheses on the Open Science Framework website at https://osf.io/zdaxy. We excluded participant data based on our pre-registered data analysis plan (see Appendix), retaining the data of 256 participants ( 139 women, mean age $=33, S D=12$ ).

\section{Materials}

The attitude object (Bob) was represented by an image of a White male (taken from the Chicago Face Database: Ma, Correll, \& Wittenbrink, 2015). Six additional faces of White males were selected for use as control stimuli in the evaluation tasks. All faces were selected on the basis of a rating study in which 51 Prolific Academic participants rated the stimuli as evaluatively neutral 
(i.e., ratings near the mid-point of the 7-point Likert scale ranging from 1 [very negative] to 7 [very positive]; Bob: $M=3.96, S D=0.96$; others: $M=[3.86-4.15], S D=[0.89-1.06])$.

Each participant was presented with a total of 5 behavioral statements about Bob: one positive, one negative, and three neutral statements. Selection of the statements was based on a prerating study in which 100 Prolific Academic participants rated 90 behavioral statements presented in compound with a picture that is related to the behavior on four characteristics: (1) the valence of the statement, (2) the valence of the picture, (3) how well they could remember the statement after cueing with the picture, and (4) how diagnostic of the person's true character they considered the statement to be (on a scale ranging from 1 [not at all diagnostic] to 5 [extremely diagnostic]). We selected two negatively valenced statements, one that was rated as high in diagnosticity $(M=4.06$, $S D=1.02)$ and one that was rated significantly lower in diagnosticity $(M=2.85, S D=0.99)$. We also selected one positively valenced statement that was rated as high in diagnosticity $(M=4.08, S D=$ $0.80)$ and one that was rated significantly lower in diagnosticity $(M=3.14, S D=0.89)$. The four valenced statements were matched on rated picture valence and ease of memory retrieval, $p \mathrm{~s}>.55$, and can be found in Appendix. Additionally, three evaluatively neutral statements were selected that were rated as low in diagnosticity $(M=[1.20-1.36], S D=[0.63-0.80])$.

\section{Procedure}

In line with recommendations by Zhou and Fishbach (2016) to prevent selective attrition, participants were first (1) informed about the duration of the experiment and (2) warned that dropping out could affect the quality of data and that it is essential for scientific advance to have good data. Next, participants provided informed consent and answered demographic questions asking for their age, gender, and country of residence. 
Evaluative learning. Participants were informed that they would learn information about a person named Bob by going through several trials in which they would see a picture of Bob together with information about a behavior in which Bob engaged. Participants were asked to read the information carefully and to try and remember it. Participants then received 5 evaluative learning task trials in which a behavioral statement was presented in the middle of the screen and the picture related to the behavior was presented above the statement (Figure 1). After 8 seconds, a prompt appeared indicating that participants could now progress to the next piece of information by pushing the space bar. Throughout the task, a picture of Bob was continuously presented at the bottom of the screen. The two valenced statements (one positive and one negative) were presented on the second and fourth trials whereas the neutral statements were presented on the odd-numbered trials. The order of the positive and negative information was counterbalanced across participants as well as whether the positive information was high in diagnosticity and the negative information was low in diagnosticity or vice versa.

Implicit and explicit evaluation. Participants next completed measures of implicit and explicit evaluations (order counterbalanced). For both tasks, participants received instructions explaining that they would complete a judgment task in which each trial involved the brief presentation of a fixation point, an image of a specific person and a Chinese character in the middle of the screen that would eventually be covered by a noisy image. For the implicit evaluation task, participants were told that they would need to indicate their liking of the Chinese characters on each trial and they were instructed not to be influenced by the images of the specific persons. For the explicit evaluation task, participants were told that they would need to indicate their liking of the specific persons on each trial and they were instructed not to be influenced by the images of the Chinese characters. 
In line with standard procedures (Payne et al., 2005), during each trial of the AMP, participants saw a prime stimulus for $75 \mathrm{~ms}$, a blank screen for $125 \mathrm{~ms}$, and a Chinese ideograph for $100 \mathrm{~ms}$, which was then covered with a black-and-white pattern mask. According with Payne et al. (2008), a prompt then appeared asking participants to rate the Chinese character (implicit evaluation task) or the specific person (explicit evaluation task) on a four-point scale ranging from 1 (very unpleasant) to 4 (very pleasant). The implicit evaluation task consisted of 60 trials, half with the face of Bob as prime and half with a control face as prime (each of the 6 control faces was presented on 5 trials). The explicit evaluation task consisted of 8 trials, one with each control face and two with the face of Bob (to allow computation of reliability). Importantly, for the whole duration of the implicit and explicit evaluation task, one picture (that was presented together with either the positive or negative behavioral statement of Bob in the learning phase) was always presented at the top of the screen (to facilitate acting as a memory retrieval cue: Van Dessel, Cone et al., 2018). Whether the positive or negative information was cued in the evaluation tasks was counterbalanced.

Additional questions. Next, participants were shown the image of Bob as well as a list of 8 valenced behavioral statements in which the two previously presented valenced statements about Bob were interspersed. Participants were asked to indicate which of the behaviors the depicted person had engaged in. Finally, participants indicated whether they knew Chinese or Mandarin and therefore recognized some of the Chinese characters they saw. They were then thanked for their participation.

\section{Results}

Implicit evaluation scores were computed by subtracting the mean pleasantness ratings of Chinese characters on implicit evaluation task trials with control faces as prime from the mean ratings in trials with the face of Bob as prime. Explicit evaluation scores were computed by subtracting the mean pleasantness ratings of control faces on explicit evaluation task trials from the 
mean ratings of Bob. For both measures, higher scores indicate a stronger preference for Bob over the other persons. Split-half reliability, calculated on the basis of an odd-even split, was $r(254)=.37$ for the implicit evaluation score and $r(254)=.57$ for the explicit evaluation score. Implicit and explicit evaluation scores correlated significantly with one another, $r(254)=.31, p<.001$.

To facilitate comparison of implicit and explicit evaluations, we standardized these scores by subtracting the mean score across participants from participants' individual scores and dividing by the standard deviation of the score. Means and standard deviations of standardized scores in each of the different manipulation conditions are reported in Table 2, unstandardized scores for Bob and other targets can be found in Appendix.

We first performed an ANOVA on standardized implicit and explicit evaluation scores that included the within-subjects factor Type of Evaluation Measure (implicit vs. explicit AMP) as well as the four between-subject factors Diagnosticity (positive vs. negative information more diagnostic), Cueing (positive vs. negative information cued), Primacy (positive vs. negative information presented first), and Task Order (implicit vs. explicit AMP performed first). We observed a main effect of Diagnosticity, $F(1,240)=31.47, p<.001, \eta^{2}=0.12$ (Table 2), which was qualified by an interaction with Type of Evaluation Measure, $F(1,240)=12.09, p<.001, \eta^{2}=0.05$. The main effects of Cueing, $F(1,240)=2.64, p=.11, \eta^{2}=0.01$, and Primacy, $F(1,240)=2.69, p=.10, \eta^{2}=0.01$, were not significant. We observed an interaction between Primacy, Type of Evaluation Measure, and Task Order, $F(1,240)=4.70, p=.031, \eta^{2}=0.02$, but no other significant interaction effects, $F \mathrm{~s}<$ $2.98, p \mathrm{~s}>.086, \eta^{2} \mathrm{~s}<0.02$.

Planned comparisons were performed to examine effects of Diagnosticity, Cueing and Primacy on implicit and explicit evaluation scores separately. First, for the effect of Diagnosticity, we observed a stronger implicit preference for Bob when the positive information was more 
diagnostic $(M=0.15, S D=0.96)$ than when the negative information was more diagnostic $(M=-$ $0.15, S D=1.02), t(254)=2.41, p=.008, d=0.30$. The Bayes Factor, computed in accordance with Rouder, Speckman, Sun, Morey, and Iverson (2009), with a prior set at $d=0.20$, indicated that the alternative hypothesis (that participants who received more diagnostic positive information exhibited a stronger implicit preference for Bob) was approximately 6 times more likely than the null hypothesis, $\mathrm{BF}_{1}=6.34$. The same pattern of results was observed on explicit evaluations (diagnostic information positive: $M=0.40, S D=0.91$; diagnostic information negative: $M=-0.40, S D=0.92$ ), but the diagnosticity effect was stronger, $t(254)=7.05, p<.001, d=0.88, \mathrm{BF}_{1}>10.000$.

Second, comparisons looking at the effect of Cueing revealed that participants exhibited a stronger implicit preference for Bob when the positive information was cued $(M=0.09, S D=1.05)$ than when the negative information was cued $(M=-0.08, S D=0.94)$, but this effect was only marginally significant, $t(254)=1.37, p=.086, d=0.17$, and the Bayes Factor indicated only slightly more evidence for the alternative compared to the null hypothesis, $\mathrm{BF}_{1}=1.32$. The effect of Cueing also did not reach standard significance levels for explicit evaluations (cued information positive: $M$ $=0.08, S D=0.96$; cued information negative: $M=-0.08, S D=1.03), t(254)=1.32, p=.093, d=$ $0.16, \mathrm{BF}_{1}=1.25$

Third, we did not observe an effect of Primacy on implicit evaluations. Participants' implicit evaluations of Bob was not significantly stronger when the positive information was presented first $(M=0.05, S D=0.87)$ than when it was presented second $(M=-0.05, S D=1.12), t(254)=0.75, p=$ $.23, d=0.09$. The Bayes Factor slightly favored the null hypothesis, $\mathrm{BF}_{0}=1.43$. In contrast, participants did exhibit a stronger explicit preference for Bob when the positive information was presented first $(M=0.13, S D=1.01)$ than when the negative information was presented first $(M=$ $0.13, S D=0.98), t(254)=2.07, p=.020, d=0.26, \mathrm{BF}_{1}=3.50$. We explored the Primacy $\mathrm{x}$ Type of 
Evaluation Measure $\mathrm{x}$ Task Order interaction that we observed in the ANOVA with a post-hoc test (the only performed test that we did not pre-register in our data analysis plan - see Appendix). We observed a stronger effect of Primacy on explicit compared to implicit evaluations only for participants who first completed the implicit evaluation task and then completed the explicit evaluation task, $F(1,115)=4.18, p=.043, \eta^{2}=0.04$, showing a primacy effect on explicit, $t(121)=$ 1.93, $p=.028, d=0.35, \mathrm{BF}_{1}=2.82$, but not implicit evaluations, $t(121)=-0.41, p=.66, d=0.07$, $\mathrm{BF}_{0}=2.52$, but no interaction effect for the other participants, $F(1,125)=0.80, p=.37, \eta^{2}<0.01$.

\section{Discussion}

Experiment 1 provided strong evidence for effects of diagnosticity on implicit and explicit evaluation evaluations, showing larger effects on explicit evaluations. These findings replicate prior work (Brannon \& Gawronski, 2017; Cone \& Ferguson, 2015). We also observed a primacy effect on explicit evaluations but no significant evidence for primacy effects on implicit evaluations or for memory cueing effects on implicit and explicit evaluations. To examine the robustness and generalizability of these results, we performed a second experiment that probed diagnosticity, memory cueing, and primacy effects as obtained on the basis of slightly different variations of the used manipulations on implicit and explicit evaluations probed with two versions of another task that is often used to measure implicit evaluations: the evaluative priming task (EPT).

\section{Experiment 2}

\section{Participants}

A total of 400 Prolific Academic participants were recruited for this study, leaving 326 participants (172 women, mean age $=36, S D=12)$ after data-exclusion (see Appendix). The preregistered plan, experiment script, data, and analysis code for Experiment 2 are available at https://osf.io/yarxs/. 


\section{Procedure}

Experiment 2 was identical to Experiment 1 except for the following points. First, we used (two variants of) the EPT rather than the AMP to measure implicit and explicit evaluations. In the implicit EPT, participants were informed that they would perform a task in which they would see a fixation point, an image of a specific person and a positive or negative word on each trial. They were instructed to categorize the words as either "Good" or "Bad" with I or E keyboard presses as quickly as possible, while making as few mistakes as possible. The task comprised 4 practice trials and 60 test trials. In line with standard procedures (Spruyt, De Houwer, Hermans, \& Eelen, 2007), a single trial consisted of the presentation of a fixation cross for $500 \mathrm{~ms}$, a blank screen for $500 \mathrm{~ms}$, a prime for $200 \mathrm{~ms}$, a post-prime interval for $50 \mathrm{~ms}$, and the presentation of a target word for a maximum of $1500 \mathrm{~ms}$. Targets consisted of 5 positive words (e.g., the word "pleasant") and 5 negative words (e.g., the word "annoying"). Prime stimuli were the picture of Bob (30 trials) and the pictures of the six other faces (30 trials: each control face 5 times). In the explicit EPT, participants were instructed to indicate their liking of the person presented before the positive or negative word. Each trial, participants first saw the prime and target word presentation and were then asked to rate their liking of the primed person on a 4-point scale from "very unpleasant" to "very pleasant" (we chose not to use a 2-point scale with only "Good" and "Bad" to prevent ceiling effects). They completed 4 practice trials and 8 test trials, one with each control face and two with the face of Bob. The order of implicit and explicit EPT administration was counterbalanced across participants and conditions.

A second adaptation compared to Experiment 1 was that Experiment 2 employed different statement pairs. An anonymous reviewer argued that the manipulation of diagnosticity in Experiment 1 might have been so strong that it over-powered the other manipulations. Hence, we now used statement pairs that differed less strongly in diagnosticity. More specifically, we used six statement 
pairs, three pairs that consisted of high diagnostic positive and low diagnostic negative information and three pairs that consisted of high diagnostic negative and low diagnostic positive information (see Appendix). Selection was based on a pre-rating study in which Prolific Academic participants provided ratings of diagnosticity and valence for 180 behavioral statements. Statements in each pair were selected to have similar ratings of valence extremity and a difference in rated diagnosticity of approximately 0.75 points. This difference in diagnosticity is substantially less than the 1.08 point mean difference in Experiment.

Finally, following suggestions by the editor, we made changes to our manipulation of cueing and primacy to provide more opportunity for observing these effects. Specifically, we now presented the first valenced behavioral statement as the first piece of information about Bob (rather than after a neutral behavioral statement) to boost the primacy effect and we presented the memory retrieval cue only when the evaluative stimuli (Bob and other person stimuli) were presented in the evaluation task (rather than for the full duration of the task) to boost the cueing effect.

\section{Results}

Implicit EPT data were dropped for trials with an incorrect response (3.57\%) and for trials in which reaction times (RTs) were at least 2.5 standard deviations removed from an individual's mean (3.12\%). Implicit evaluation scores were computed by (1) subtracting the subjects' mean RT in trials with positive targets and Bob primes from their mean RT in trials with negative targets and Bob primes, (2) subtracting subjects' mean RT in trials with positive targets and other person primes from their mean RT in trials with negative targets and other person primes, and (3) subtracting the second from the first difference score. Explicit evaluation scores were computed by subtracting the mean pleasantness ratings of control faces from the mean ratings of Bob. Split-half reliability was $r(324)=$ 
.15 for the implicit evaluation score and $r(324)=.38$ for the explicit evaluation score. Implicit and explicit evaluation scores correlated significantly, $r(324)=.11, p=.041$.

The ANOVA on standardized implicit and explicit evaluation scores (Table 2) that included the factor Type of Evaluation Measure (implicit vs. explicit evaluation) as well as the 4 betweensubjects factors Diagnosticity, Cueing, Primacy, and Task Order, revealed a main effect of Diagnosticity, $F(1,310)=11.70, p<.001, \eta^{2}=0.04$, and a main effect of Primacy, $F(1,310)=5.19$, $p=.023, \eta^{2}=0.02$. We did not observe a main effect of Cueing, $F(1,310)=0.84, p=.34, \eta^{2}<0.01$, or any interaction effects, $F \mathrm{~s}<2.88, p \mathrm{~s}>.090, \eta^{2} \mathrm{~s}<0.01$.

Planned comparisons testing the effect of Diagnosticity revealed a stronger implicit preference for Bob when the positive information was more diagnostic $(M=0.10, S D=1.06)$ than when the negative information was more diagnostic $(M=-0.10, S D=0.93), t(324)=1.77, p=.039$, $d=0.20, \mathrm{BF}_{1}=2.15$ (Table 2). Importantly, this is the first demonstration of the effects of diagnosticity on implicit evaluations on a measure other than the AMP. The same pattern of results was observed on explicit evaluations (diagnostic information positive: $M=0.18, S D=0.92$; diagnostic information negative: $M=-0.18, S D=1.05), t(324)=3.25, p<.001, d=0.36, \mathrm{BF}_{1}=$ 44.30.

Second, comparisons testing the effect of Cueing did not show a significant effect on implicit evaluations (cued information positive: $M=0.00, S D=0.97$; cued information negative: $M=-0.01$, $S D=1.04), t(324)=0.04, p=.49, d=0.01, \mathrm{BF}_{0}=2.69$. We also did not observe an effect of Cueing on explicit evaluations (cued information positive: $M=0.03, S D=0.93$; cued information negative: $M=-0.04, S D=1.08), t(324)=0.65, p=.26, d=0.07, \mathrm{BF}_{0}=1.67$

Third, comparisons revealed a significant effect of Primacy on implicit evaluations. Participants' implicit preference for Bob was stronger when the positive information was presented 
first $(M=0.09, S D=0.97)$ than when it was presented second $(M=-0.10, S D=1.02), t(324)=1.70$, $p=.046, d=0.19, \mathrm{BF}_{1}=1.94$. The effect of Primacy on explicit evaluations was not significant (first presented information positive: $M=0.07, S D=1.01$; first presented information negative: $M=-0.07$, $S D=0.99), t(324)=1.31, p=.095, d=0.15, \mathrm{BF}_{1}=1.18$.

\section{General Discussion}

The current study investigated the moderating role of information primacy, diagnosticity and cueing in effects of valenced information on implicit and explicit evaluations as measured with procedures that were matched on factors unrelated to the constructs of interest (Payne et al., 2008). The results offer important information about these moderators. First, our results provide strong evidence that diagnosticity moderates effects of valenced information not only on explicit but also on implicit evaluations. This accords with recent evidence (Cone \& Ferguson, 2015) and extends it by showing the moderating effect of information diagnosticity (1) in an alternative evaluative learning paradigm (e.g., involving only two matched behavioral statements rather than a large set of statements), (2) on more tightly matched implicit and explicit evaluation measures, (3) using two different measures of implicit evaluations (not only the AMP but also the EPT), and (4) in comparison to other moderators.

Second, results provide only weak evidence for the idea that primacy of valenced information is an important moderator of effects on implicit evaluation, which contrasts with previous findings (e.g., Gregg et al., 2006; see below for a comparison of our study to the Gregg et al. study). Specifically, Experiment 1 found weak evidence for the absence of an effect of primacy on implicit evaluation and Experiment 2 found weak evidence for the presence of a small effect. These findings are notable because the order in which one encounters valenced information is often considered an important determinant of subsequent affect, construal, or subjective feelings of diagnosticity which 
are sometimes thought to influence implicit evaluation (see Cone et al., 2017). Results provided stronger evidence for the presence of a primacy effect on explicit evaluation, which accords with some prior research (see Asch, 1946; Miller \& Campbell, 1959), but this effect was observed only in Experiment 1.

Third, in contrast to a recent study (Van Dessel, Cone, et al., 2018), we did not find evidence for an effect of memory cueing on implicit and explicit evaluation. One explanation is that this is due to a ceiling effect in the current study. Different to the experiments reported here, the study by Van Dessel, Cone et al. (2018) included two target persons as attitude stimuli (Bob and Jake) and four pieces of valenced information. It is possible that cueing more strongly facilitates memory retrieval in a context where more pieces of valenced information have been presented.

It is also of interest that we did not observe any interactions of the three information characteristics on implicit or explicit evaluation. For instance, one might expect that primacy more strongly influences evaluations when the first presented information is highly diagnostic because participants then immediately discard the second information. Similarly, an interaction of cueing and diagnosticity might be expected such that cueing of low but not high diagnostic information influences evaluation (e.g., due to a ceiling effect). Given the small (and non-significant) effects of primacy and cueing overall, however, the absence of interaction effects could also be due to a lack of power to observe effects smaller than $\eta^{2}=.02$.

\section{Comparison of effects on implicit and explicit evaluations}

Importantly, the current study also provides information about the differential effect of information characteristics on implicit and explicit evaluations. We observed that, compared to effects on explicit evaluations, all effects on implicit evaluations were small (all $d$ s $<0.32$ ). The reason for this might be that unintentional evaluation is more indirectly related to the evaluation of 
the target stimulus and variance in observed scores therefore more strongly reflects other factors (such as genuine liking of some of the Chinese characters in the AMP or the target words in the EPT). This might accord with the observation that implicit evaluation scores were lower in reliability than explicit evaluation scores (although lower reliability can also indicate reduced true score rather than error score variance: De Schryver, Hughes, Rosseel, \& De Houwer, 2015). It is worth noting that, whereas diagnosticity and primacy effects were significantly reduced for implicit compared to explicit evaluations in Experiment 1, this was not the case in Experiment 2. This might relate to the fact that effects were smaller in Experiment 2 in both the implicit and explicit version of the EPT (e.g., due to lower sensitivity of the EPT, weaker manipulations, or sample characteristics) which reduces the statistical power to observe moderation of the effects.

\section{Theoretical implications}

The current results are consistent with some but not all of the predictions derived from an inferential account (Van Dessel, Hughes, \& De Houwer, 2018). From this perspective, both implicit and explicit evaluations result from evaluative inferences that are made on the basis of information that is available in memory. Because information diagnosticity might be considered an index of the extent to which information allows inferences of stimulus valence, the inferential account predicted that information diagnosticity would constitute the strongest moderator of valenced information effects on both implicit and explicit evaluation. This was observed in both experiments. The fact that Experiment 2 found an effect of diagnosticity on an implicit evaluation measure with low reliability even though the difference in rated diagnosticity was less than 1 point on a 5-point scale provides strong evidence that fits the idea that not only explicit but also implicit evaluation is very strongly dependent on diagnosticity. Results also confirm the prediction that diagnosticity should have a stronger effect on explicit than on implicit evaluation (because the measurement context more 
strongly facilitates the validation information on the basis of diagnosticity) but only in Experiment 1 . The prediction that the manipulation of memory encoding of information on the basis of primacy and of memory retrieval on the basis of cueing should also influence implicit and explicit evaluations did not find clear support in the current results. Results were inconclusive about the effects of both manipulations, though the evidence for an effect of Primacy was stronger.

We also specified predictions of a dual-process account of evaluation (i.e., the systems of reasoning account: Rydell \& McConnell, 2006). From this perspective, propositional learning processes (such as inferential reasoning on the basis of diagnosticity) determine explicit evaluation whereas automatic associative learning processes (such as automatic association formation on the basis of pairings of stimuli and valenced events) determine implicit evaluation. Overall, results contrast with predictions derived from this account because (1) information primacy did not influence implicit evaluations more strongly than explicit evaluations and (2) information diagnosticity strongly influenced not only explicit but also implicit evaluations. The former observation contrasts with the idea that implicit evaluations reflect the activation of associations that, once established, cannot be readily changed, as supported by studies showing an insensitivity of implicit evaluations to counter-attitudinal information (e.g., Gregg et al., 2006). One explanation for the observed discrepancy with previous findings might be that previous findings did not reflect the insensitivity of implicit evaluations to counter-attitudinal information (due to the robustness of learned associations that underlie implicit evaluations) but rather the sensitivity of implicit evaluations to other factors such as the extensiveness of information processing (see Wyer, 2016). For instance, in the Gregg et al. (2006) studies there was more opportunity to process the information that was presented first which might have facilitated automatic evaluative inferences on the basis of this information. The fact that the evaluative learning procedure and valenced information were more 
tightly matched in the current study might explain why we did not find a similar implicit-explicit dissociation on the basis of information primacy.

Even though the current results are not in line with the predictions of a systems of reasoning account (Rydell \& McConnell, 2006), this does not mean that they cannot be accommodated by dualprocess theories. McConnell and Rydell (2014) have argued that (1) the distinct systems underlying implicit and explicit evaluation are not necessarily entirely unresponsive to the type of information that the other system employs and (2) there is not always a one-to-one correspondence between evaluation measures and systems. This raises the possibility that evaluations as captured by implicit measures can be influenced by information diagnosticity even if they are in part driven by a slowlearning, associative, system. The Associative-Propositional Evaluation Model (APE Model: Gawronski \& Bodenhausen, 2006) makes it even more explicit that there is opportunity for an immediate influence of propositional reasoning processes on implicit evaluations because it postulates that propositional processes can readily influence the associative processes underlying implicit evaluations. For instance, information that is considered highly diagnostic might strongly facilitate the activation of evaluative associations that are congruent with this information and thereby influence implicit evaluations.

\section{Practical implications and future directions}

The current research provides one of the first demonstrations that the examination of the moderators of implicit and explicit evaluation as measured in matched tasks can provide important information about the underpinnings of implicit and explicit evaluation. However, the current results also have practical implications. For instance, they clearly indicate that, in order to change implicit evaluations, it might be most useful to provide information that is high in diagnosticity. This approach is not often taken in intervention research that aims to modify (unwanted) implicit 
evaluations. Yet, recent studies that have used diagnostic information to change implicit evaluations of well-known stimuli (e.g., of cigarettes: Smith \& De Houwer, 2015; of a well-known historic figure: Van Dessel, Ye, \& De Houwer, 2018) support this approach.

We hope that future research will further examine moderators of implicit and explicit evaluations in high-powered, comparative studies that control for measurement-related issues. This type of research cannot only probe moderating effects of information characteristics related to the information content but also related to the source of the information (e.g., source credibility: Smith, De Houwer, \& Nosek, 2013) or the persuasive context (e.g., elaboration instructions: Wyer, 2016) and their interactions (e.g., the moderating effect of elaboration opportunity on primacy effects: Haugtvedt \& Wegener, 1994). These studies could also probe differences related to the type of evaluative stimuli (e.g., novel versus well-known stimuli) and compare effects on different evaluation measures such as on AMPs (or IATs, EPTs) that measure evaluations under different automaticity conditions (e.g., not only intentionality but also rapidity or cognitive load). This approach might help us to obtain a clear overview of the moderators that influence implicit and explicit evaluation and the extent of moderation, allowing stronger conclusions about when and why implicit and explicit evaluations dissociate. Such information might be crucial to get a better insight into how implicit and explicit evaluations relate to real-life behavior and how implicit (and explicit) evaluation measures can be of use to researchers and practitioners. 


\section{References}

Allport, G. (1935). Attitudes. In Murchison, C. (Ed.). A Handbook of Social Psychology (pp. 789843). Worcester, MA: Clark University Press.

Asch, S. E. (1946) Forming impressions of personality, Journal of Abnormal and Social Psychology, $41,258-290$.

Banaji, M., and A. Greenwald. 2013. Blindspot: Hidden biases of good people. New York: Delcorte Press.

Cameron, C. D., Brown-Iannuzzi, J. L., \& Payne, B. K. (2012). Sequential priming measures of implicit social cognition: A meta-analysis of associations with behavior and explicit attitudes. Personality and Social Psychology Review, 4, 330-350.

Cone, J., \& Ferguson, M. J. (2015). He Did what?: The Role of Diagnosticity in Revising Implicit evaluations. Journal of Personality and Social Psychology, 108, 37-57. doi:10.1037/pspa0000014

Cone, J., Mann, T. C., \& Ferguson, M. J. (2017). Can we change our implicit minds? New evidence for how, when, and why implicit impressions can be rapidly revised. Advances in Social Psychology.

Corneille, O., \& Stahl, C. (2018). Associative Attitude Learning: A Closer Look at Evidence and how it Relates to Attitude Models. Personality and Social Psychology Review.

De Houwer, J. (2014). A Propositional Model of Implicit Evaluation. Social and Personality Psychology Compass, 8, 342-353.

De Houwer, J., Thomas, S., \& Baeyens, F. (2001). Associative learning of likes and dislikes: A review of 25 years of research on human evaluative conditioning. Psychological Bulletin, $127,853-869$. 
De Schryver, M., Hughes, S., Rosseel, Y., \& De Houwer, J. (2015). Unreliable Yet Still Replicable: A Comment on LeBel and Paunonen (2011). Frontiers in Psychology. 6: 2039.

Fazio, R. H., Sanbonmatsu, D. M., Powell, M. C., \& Kardes, F. R. (1986). On the automatic activation of attitudes. Journal of Personality and Social Psychology, 50, 229-238.

Friese, M., Hofmann, W., \& Schmitt, M. (2009). When and why do implicit measures predict behaviour? Empirical evidence for the moderating role of opportunity, motivation, and process reliance. European Review of Social Psychology, 19, 285-338.

Gawronski, B., \& Brannon, S. M. (2018). Attitudes and the implicit-explicit dualism. In D. Albarracín \& B. T. Johnson (Eds.), Handbook of attitudes (2nd edition). New York: Taylor \& Francis.

Gawronski, B., \& Bodenhausen, G. V. (2006). Associative and propositional processes in evaluation: An integrative review of implicit and explicit attitude change. Psychological Bulletin, 132, 692-731.

Gawronski, B., \& De Houwer, J. (2014). Implicit measures in social and personality psychology. In H. T. Reis, \& C. M. Judd (Eds.), Handbook of research methods in social and personality psychology (2nd edition, pp. 283-310). New York: Cambridge University Press.

Greenwald, A. G., McGhee, D. E., \& Schwartz, J. L. (1998). Measuring individual differences in implicit cognition: the implicit association test. Journal of Personality and Social Psychology, $74,1464-1480$.

Gregg, A. P., Seibt, B., \& Banaji, M. R. (2006). Easier done than undone: asymmetry in the malleability of implicit preferences. Journal of Personality and Social Psychology, 90, 1-20.

Haugtvedt, C. P., \& Wegener, D. T. (1994). Message Order Effects in Persuasion: An Attitude Strength Perspective. Journal of Consumer Research, 21, p. 205-218 
Heycke, T., Gehrmann, S., Haaf, J.M., \& Stahl, C. (2018). Of two minds or one? A registered replication of Rydell et al. (2006). Cognition and Emotion.

Hofmann, W., De Houwer, J., Perugini, M., Baeyens, F., \& Crombez, G. (2010). Evaluative conditioning in humans: a meta-analysis. Psychological Bulletin, 136, 390-421.

Hu, X., Gawronski, B., \& Balas, R. (2017). Propositional versus dual-process accounts of evaluative conditioning: I. The Effects of Co-Occurrence and Relational Information on Implicit and Explicit Evaluations. Personality and Social Psychology Bulletin, 43, 43, 17-32.

Ma, D.S., Correll, J. \& Wittenbrink, B. (2015). The Chicago Face Database: A Free Stimulus Set of Faces and Norming Data. Behavior Research Methods, 47, 1122-1135.

Mandelbaum, E. (2016). Attitude, inference, association: On the propositional structure of implicit bias. Noûs, 50, 629-658.

Mann, T. C., \& Ferguson, M. J. (2015). Can we undo our first impressions? The role of reinterpretation in reversing implicit evaluations. Journal of Personality and Social Psychology, 108, 823-849.

Mann, T. C., \& Ferguson, M. J. (2017). Reversing implicit first impressions through reinterpretation after a two-day delay. Journal of Experimental Social Psychology, 68, 122-127.

McConnell, A. R., \& Rydell, R. J. (2014). The Systems of Evaluation Model: A dual-systems approach to attitudes. In J. W. Sherman, B. Gawronski, \& Y. Trope (Eds.), Dual process theories of the social mind (pp. 204-217). New York: Guilford.

Miller, N. \& Campbell, D.T. (1959), Recency and primacy in persuasion as a function of the timing of speeches and measurements. Journal of Abnormal and Social Psychology, 59, 1-9. 
Olson, M. A., \& Fazio, R. H. (2004). Reducing the influence of extra-personal associations on the Implicit Association Test: Personalizing the IAT. Journal of Personality and Social Psychology, 86, 653-667.

Payne, B. K,. Burkley, M. A., \& Stokes, M. B. (2008). Why Do Implicit and Explicit Attitude Tests Diverge? The Role of Structural Fit. Journal of Personality and Social Psychology, 94, 1631.

Payne, B. K., Cheng, C. M., Govorun, O., \& Stewart, B. D. (2005). An inkblot for attitudes: Affect misattribution as implicit measurement. Journal of Personality and Social Psychology, 89, 277-293.

Petty, R. E., \& Brinol, P. (2009). Implicit ambivalence: A meta-cognitive approach. In R. E. Petty, R. H. Fazio, \& P. Brinol (Eds.), Attitudes: Insights from the new implicit measures (pp. 119161). New York: Psychology Press.

Rothermund, K., \& Wentura, D. (2004). Underlying processes in the Implicit Association Test (IAT): Dissociating salience from associations. Journal of Experimental Psychology: General, 133, 139-165.

Rouder, J. N., Speckman, P. L., Sun, D., Morey, R. D., \& Iverson, G. (2009). Bayesian t tests for accepting and rejecting the null hypothesis. Psychonomic Bulletin \& Review, 16, 225-237.

Rydell, R. J., \& McConnell, A. R. (2006). Understanding implicit and explicit attitude change: A systems of reasoning analysis. Journal of Personality and Social Psychology, 91, 995- 1008.

Smith, C. T., \& De Houwer, J. (2015). Hooked on a feeling: Affective anti-smoking messages are more effective than cognitive messages at changing implicit evaluations of smoking. Frontiers in Psychology, 6:1488. 
Smith, C. T., De Houwer, J., \& Nosek, B. (2013). Consider the source: persuasion of implicit evaluations is moderated by source credibility. Personality and Social Psychology Bulletin, 39, 193-205.

Van Dessel, P., Cone, J. Gast, A., \& De Houwer, J. (2018). The important role of memory processes in implicit evaluation. Manuscript in preparation. Project available at: https://osf.io/2by8c/

Van Dessel, P., De Houwer, J., Gast, A., Smith, C. T., \& De Schryver, M. (2016). Instructing Implicit Processes: When Instructions to Approach or Avoid Influence Implicit but not Explicit Evaluation. Journal of Experimental Social Psychology, 63, 1-9.

Van Dessel, P., Hughes, S., \& De Houwer, J. (2018). How Do Actions Influence Attitudes? An Inferential Account of the Impact of Action Performance on Stimulus Evaluation. Personality and Social Psychology Review.

Van Dessel, P., Ye, Y., \& De Houwer, J. (2018). Changing Deep-rooted Implicit Evaluation in the Blink of an Eye: Negative Verbal Information Shifts Automatic Liking of Gandhi. Social Psychological and Personality Science.

Wyer, N. A. (2016). Easier done than undone... by some of the people, some of the time: The role of elaboration in explicit and implicit group preferences. Journal of Experimental Social Psychology, 63, 77-85.

Zhou, H., \& Fishbach, A. (2016). The Pitfall of Experimenting on the Web: How Unattended Selective Attrition Leads to Surprising (Yet False) Research Conclusions. Journal of Personality and Social Psychology, 11, 493-504. 


\section{Tables}

Table 1. Means and standard deviations for standardized implicit and explicit evaluation scores for participants in the different manipulation conditions of Experiment 1.

\begin{tabular}{cccccc}
\hline & $\mathrm{N}$ & \multicolumn{2}{c}{ Implicit evaluation scores } & \multicolumn{2}{c}{ Explicit evaluation scores } \\
\hline Positive info condition: & & Mean & SD & Mean & SD \\
\hline First, more diagnostic, cued & 32 & 0.14 & 0.80 & 0.51 & 0.69 \\
Last, more diagnostic, cued & 33 & 0.43 & 1.11 & 0.47 & 1.00 \\
First, less diagnostic, cued & 28 & -0.07 & 0.97 & -0.26 & 0.98 \\
Last, less diagnostic, cued & 32 & -0.17 & 1.22 & -0.44 & 0.78 \\
First, more diagnostic, not cued & 35 & 0.03 & 0.93 & 0.53 & 1.02 \\
Last, more diagnostic, not cued & 28 & -0.02 & 0.98 & 0.04 & 0.83 \\
First, less diagnostic, not cued & 33 & 0.08 & 0.79 & -0.34 & 0.98 \\
Last, less diagnostic, not cued & 35 & -0.41 & 1.02 & -0.55 & 0.78 \\
\hline
\end{tabular}


Table 2. Means and standard deviations for standardized implicit and explicit evaluation scores for participants in the different manipulation conditions of Experiment 2.

\begin{tabular}{cccccc}
\hline & $\mathrm{N}$ & \multicolumn{2}{c}{ Implicit evaluation scores } & \multicolumn{2}{c}{ Explicit evaluation scores } \\
\hline Positive info condition: & & Mean & SD & Mean & SD \\
\hline First, more diagnostic, cued & 40 & 0.17 & 1.08 & 0.25 & 0.82 \\
Last, more diagnostic, cued & 41 & -0.04 & 0.95 & 0.12 & 0.78 \\
First, less diagnostic, cued & 43 & 0.06 & 0.95 & -0.11 & 1.01 \\
Last, less diagnostic, cued & 50 & -0.15 & 0.88 & -0.08 & 1.04 \\
First, more diagnostic, not cued & 44 & 0.15 & 0.98 & 0.32 & 0.98 \\
Last, more diagnostic, not cued & 39 & 0.10 & 1.24 & 0.01 & 1.06 \\
First, less diagnostic, not cued & 39 & -0.03 & 0.87 & -0.18 & 1.13 \\
Last, less diagnostic, not cued & 30 & -0.33 & 1.02 & -0.44 & 1.04 \\
\hline
\end{tabular}




\section{Figures}

\section{Figure 1}

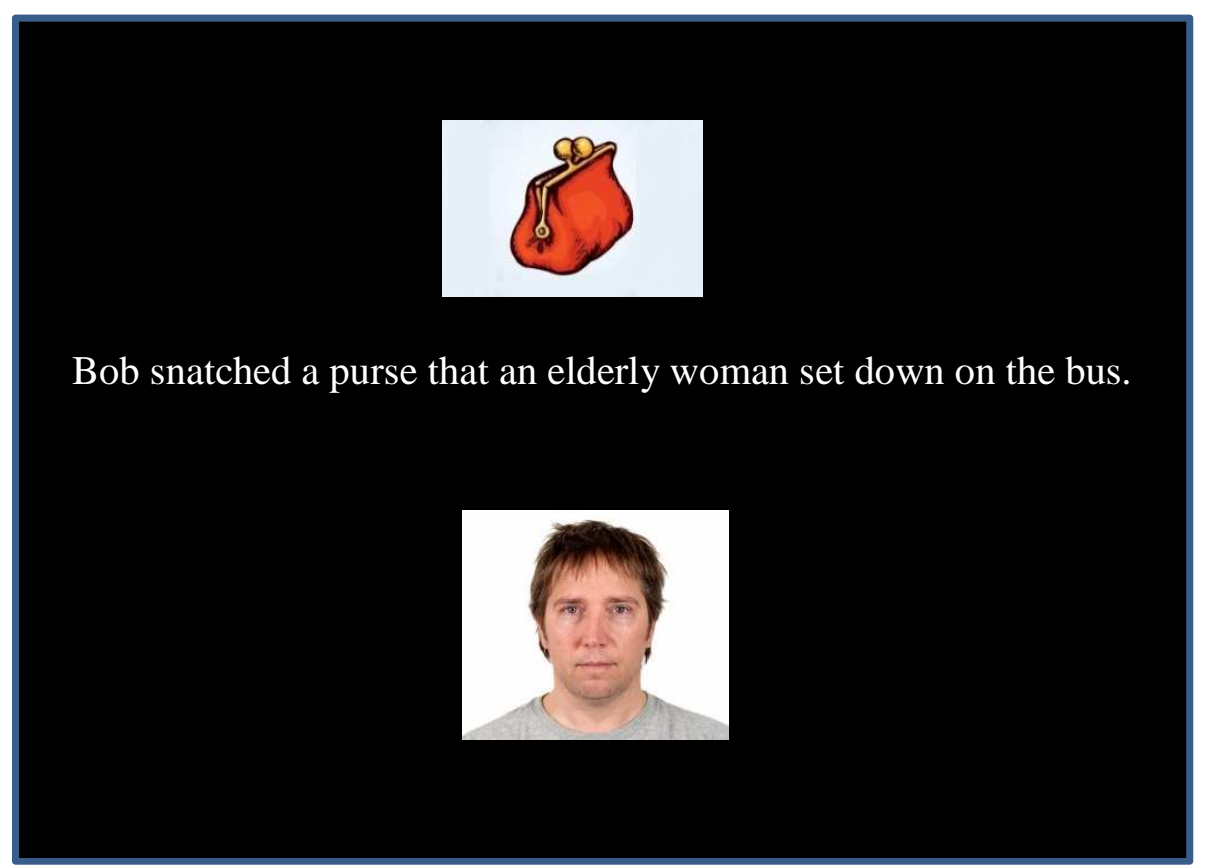




\section{Figure captions}

Figure 1. Illustration of an evaluative learning trial with high diagnostic negative information in Experiment 1. 